\title{
A comparison between applications of the Lyapunov's second (direct) method and fixed point theory
}

\author{
Sadeq Taha Abdulazeez ${ }^{\mathrm{a}, *}$, Sizar Abid Mohammed ${ }^{\mathrm{b}}$, Mehmut MODANLI $^{\mathrm{c}}$ \\ ${ }^{a}$ Department of Mathematics-College of Basic Education, University of Duhok, Iraq, Duhok. \\ ${ }^{b}$ Department of Mathematics-College of Basic Education, University of Duhok, Iraq, Duhok. \\ ${ }^{c}$ Department of Mathematics- Faculty of Arts and Sciences, Harran University, Sanliurfa, Turkey.
}

\begin{abstract}
In this article, we will discuss the application of the Lyapunov's second method and fixed point theories to certain differential equations of first and second order. First, we will introduce some basic information about these subjects, and later, we give their applications concerning some specific attitude of Solutions of Delay Differential Equations. We will also do a comparison between them. Include keywords, mathematical subject classification numbers as needed. (c)2019 All rights reserved.
\end{abstract}

Keywords: Lyapunov's function, Sfixed point, stability, Delay Differential Equations.

2010 MSC: MSC 37C25, MSC 37B25, .

\section{Introduction}

For contracts, the second Lyapunov method was highly effective in stability, instability and borders, global presence, etc. However, there is a host of problems that have not been effective (see, Burton [3]). Perhaps, the reason for this fact is that the construction or identification of appropriate Lyapunov's functions that product important results for the specific behaviors mentioned in natural and functional differential and integral equations remains an open problem in literature by this time. Many authors studied specific problems that raised large hardness in the aforesaid topics and explored the specific manner of solutions over different notion about fixed points. Moreover, while the Lyapunov second method usually requires prudent conditions, the fixed point theory needs intermediate conditions. In addition, the fixed point theory can be utilized straightway to study an equation when solutions are behold at a particular interval. There are many doings achieved on the specific conduct of mentioned differential equations, According to Lyapunov second method fixed point theories, readers can judge Becker and Burton's books or papers [1], Burton [11, 2, 3, 4, 5, 6, 7], Burton and Furumochi [8], Burton and Hering [9], Burton and Townsend [10], Chen et al. [12], Cherkas and Malysheva [13], Hou and Wu [14], Jin [15], Jitsuro and Yusuke [16], Liu and Huang [18], Npoles Valds [36], Krasovskii [17], Pi [19], Sugie and Amano [20], Tun [21, 22, 23, 24? , 25, 26, 27, 28], Tun and mohammed [29, 30, 31, 32, 33, 34], Tun and Tun [35], Yoshizawa [31] Zhang ([38], [40]) and Zhou and Jiang [39]. Separate text sections with

\footnotetext{
${ }^{*}$ Corresponding author

Email addresses: sadiq. taha@uod. ac (Sadeq Taha Abdulazeez), sizar@uod.ac (Sizar Abid Mohammed), mmodanli@harran.edu.tr (Mehmut MODANLI)
}

doi:10.31559/glm2019.7.2.3 
In 2005, said Burton [5] equation type Linard with a record number with constant delay, $(L>0)$ :

$$
x^{\prime \prime}+f\left(t, x, x^{\prime}\right) x^{\prime}+b(t) g(x(t-L))=0
$$

Burton [5] earned on the terms of each solution $x(t)$ to satisfy $x(t) \rightarrow 0$ and $x^{\prime}(t) \rightarrow 0$ as $t \rightarrow \infty$ using deflation mapping. in 2011, PI [19] Discuss the characteristics of the stability solutions for the Linard type functional equation with a standard number with variable delay, $\tau(t)>0$ :

$$
x^{\prime \prime}+f\left(t, x, x^{\prime}\right) x^{\prime}+b(t) g(x(t-\tau(t)))=0 .
$$

$\mathrm{Pi}$ [19] has got some interesting conditions that guarantee that the solution is zero from Eq. (2) Stable and asymptotic using fixed point theory. On the other hand, according to our literary knowledge, the qualitative behavior of Linard type equations of the model

$$
x^{\prime \prime}+\left\{f(x)+g(x) x^{\prime}\right\} x^{\prime}+h(x)=e^{x}
$$

Their modified species have been studied by numerous researches since 1945 till now, these qualitative equations have played very important roles in many scientific fields (for instead see, Burton ([11], [2], [7]), Burton and Townsend [13], Chen et al. [12], Hou and Wu [14], Jin [15], Jitsoro and Yusoke [16], Liu and Huang [18], Npoles Valds [36], Krasovskii [17], Pi [19], Tun ([26], [28]), Tun and mohammed ([29],[31],[32], Tun and Tun [35], Yoshizawa [37], Zhang [38], Zhou and Jiang [39]and the references cited in these works.

\section{Some Basic Information}

In this section, we first give some basic information about the presence and uniqueness solutions of ordinary differential equations.

Definition 2.1. Let $f$ be a function such that $f: R \rightarrow R$. The function $f(u)$ is satisfy a Lipschitz condition on the interval $[a, b]$ if $\exists k>0$ S.T

$$
\left|f\left(u_{1}\right)-f\left(u_{2}\right)\right| \leq k\left|u_{1}-u_{2}\right|, u_{1}, u_{2} \in R .
$$

Theorem 2.2. If $g(t, u)$ and $\frac{\partial g}{\partial u}$ are the continuous of $t$ and $u$ in the region $R(a, b):\left|t-t_{0}\right| \leq a, \quad\left|u-u_{0}\right| \leq$ $b$, then there exists a unique solution $u(t)$ to the initial value problem.

$u^{\prime}=g(t, u), \quad u\left(t_{0}\right)=u_{0}$, on the some interval $\left|t-t_{0}\right| \leq h \leq a$, where $h$ is a sufficiently small positive constant.

It should be noted that the linear initial value problem

$$
u^{\prime}=a(t) u+b(t), \quad u\left(t_{0}\right)=u_{0},
$$

has a unique solution on the whole interval $\left|t-t_{0}\right| \leq h$. In other words, there is no apparent relationship between the region where the function $g(t, u)$ is continuous and the interval of existence of the solution.

Theorem 2.3. Assume that $f(t, x)$ is continuous on $B_{0}: t_{0} \leq t \leq t_{0}+a,\left\|x-x_{0}\right\| \leq b$, where both $a$ and $b$ are positive real numbers, and satisfies the Lipschitz condition in $B_{0}$. Let

$$
M=\underset{(t, x) \in B_{0}}{\max \|f(t, x)\|}, \alpha=\min \left(a, \frac{b}{M}\right) .
$$

Then, the initial value problem has unique solution $x(t)$ on $\left[t_{0}, t_{0}+\alpha\right]$.

Let us consider the system of differential equations with initial condition

$$
x_{i}^{\prime}=f_{i}\left(t, x_{1}, x_{2}, \ldots, x_{n}\right), \quad x_{i}\left(t_{0}\right)=x_{i 0},
$$

where $i=1,2, \ldots, n$. In vector notation, the above equations written as follows

$$
\left.\begin{array}{c}
x^{\prime}=f(t, x) \\
x\left(t_{0}\right)=x_{0}
\end{array}\right]
$$


. where $x=\left(x_{1}, x_{2}, \ldots, x_{n}\right), f=\left(f_{1}, f_{2}, \ldots, f_{n}\right)$, and $x_{0}=\left(x_{10}, x_{20}, \ldots, x_{n o}\right)$ are vectors in $R^{n}$. We shall assume that $f \in C\left[\Omega, R^{n}\right]$, where $\Omega$ is an open $(t, x)$-set in $R^{n+1}$.

Definition 2.4. A function $v(t)$ is called a continuation of the solution $u(t)$ to $I_{1} \supset I$ if $v(t)$ is defined on the interval $I_{1}, v(t) \equiv u(t)$ on $I$, and $v(t)$ satisfies the initial value problem $u^{\prime}=g(t, u), u\left(t_{0}\right)=u_{0}$ on $I_{1}$.

Definition 2.5. A metric space $(X, d)$ is complete if every Caochy seqeance in $X$ is convergent to an aliment in $X$.

In Math, the fixed point of a function is a point in a function field that is assigned to the function alone. Sometimes a set of elements is called a fixed set. That is, $\mathrm{c}$ is the fixed point of the function $f(x)$ iff $f(c)=c$. For example, if $f$ defined on $\mathrm{R}$ by

$$
f(x)=x^{2}-3 x+4
$$

then 2 is a fixed point of $f$, because $f(2)=2$.

Many different kinds of problems can be solved by means of fixed point theory. Generally, to solve a problem with fixed point theory is to find:

(a) a set $S$ consisting of points which would be acceptable solutions;

(b) a mapping $P: S \rightarrow S$, with the property that a fixed point solves the problem;

(c) a fixed point theorem stating that this mapping on this set will have a fixed point.

Theorem 2.6. If $\phi:(S, d) \rightarrow(S, d)$ is a contraction mapping and if $(S, d)$ is complete metric sparse, then $\phi$ has aunique fixed point; that's, there is a unique $s^{*} \in S$, such that $\phi\left(\mathrm{s}^{*}\right)=\mathrm{s}^{*}$.

Definition 2.7. The solution $x(t)$ of EQ.(4) it said to be stable if for each $\varepsilon>0$, there exists a $\delta=$ $\delta\left(t_{0}, \varepsilon\right)>0$ such that, for any solution $x(t)=x\left(t, t_{0}, x_{0}\right)$ of EQ.(4), the inequality $\left\|\bar{x}_{0}-x_{0}\right\| \leq \delta$ implies $\|\bar{x}(t)-x(t)\|<\varepsilon$ for all $t \geq t_{0}$.

Example 2.8. Consider the initial value problem

$$
x^{\prime \prime}+3 x^{\prime}+2 x=0, \quad x(0)=1, \quad x^{\prime}(0)=-3 .
$$

Let $x=x_{1}, \quad x_{1}^{\prime}=x_{2}$. Hence, we have

$$
x_{1}^{\prime}=x_{2}, x_{2}^{\prime}=-2 x_{1}-3 x_{2} .
$$

It follows that

$$
\begin{gathered}
x_{1}(t)=c_{1} e^{-t}+c_{2} e^{-2 t}, \quad t \geq 0 \\
x_{2}(t)=-c_{1} e^{-t}-2 c_{2} e^{-2 t} .
\end{gathered}
$$

When we use the above initial conditions, we get

$$
\begin{aligned}
& x_{1}(t)=-e^{-t}+2 e^{-2 t} \\
& x_{2}(t)=e^{-t}-4 e^{-2 t} .
\end{aligned}
$$

Let us apply the definition of the stability. When

$$
\left\|\left(\begin{array}{c}
x_{1}(0) \\
x_{2}(0)
\end{array}\right)-\left(\begin{array}{c}
\bar{x}_{1}(0) \\
\bar{x}_{2}(0)
\end{array}\right)\right\|=\left\|\left(\begin{array}{c}
1 \\
-3
\end{array}\right)-\left(\begin{array}{l}
0 \\
0
\end{array}\right)\right\| \leq 4<\delta,
$$

then it follows that

$$
\begin{gathered}
\left\|\left(\begin{array}{c}
x_{1}(t) \\
x_{2}(t)
\end{array}\right)-\left(\begin{array}{c}
0 \\
0
\end{array}\right)\right\|=\left\|\left(\begin{array}{c}
-e^{-t}+2 e^{-2 t} \\
e^{-t}-4 e^{-2 t}
\end{array}\right)-\left(\begin{array}{l}
0 \\
0
\end{array}\right)\right\| \\
\leq\left|-e^{-t}\right|+\left|2 e^{-2 t}\right|+\left|e^{-t}\right|+\left|-4 e^{-2 t}\right| \\
\leq 1+2+1+4<2 \delta=\varepsilon, \quad \delta=\varepsilon / 2 .
\end{gathered}
$$


This implies the zero solution of the equation is stable.

Definition 2.9. The solution $x(t)$ of EQ.(4) is said to be uniformly stable if for each $\varepsilon>0$, there exists a $\delta=\delta(\varepsilon)>o$ such that, for any solution $\bar{x}(t)=x\left(t, t_{0}, \bar{x}_{0}\right)$ of EQ. (4), the inequalities $t_{1} \geq t_{0}$ and $\left\|\bar{x}\left(t_{1}\right)-x\left(t_{1}\right)\right\| \leq \delta$ imply $\|\bar{x}(t)-x(t)\|<\varepsilon$ for all $t \geq t_{1}$.

Example 2.10. Consider the initial value problem

$$
x^{\prime \prime}+x=o, x(o)=c_{1}, \quad x^{\prime}(o)=c_{2} .
$$

Let $x=x_{1}, \quad x_{1}^{\prime}=x_{2}$. Hence, we have

$$
x_{1}^{\prime}=x_{2}, \quad x_{2}^{\prime}=-x_{1}
$$

It follows that

$$
\begin{gathered}
x_{1}(t)=c_{1} \cos t+c_{2} \sin t, \quad t \geq 0 \\
x_{2}(t)=-c_{1} \sin t+c_{2} \cos t .
\end{gathered}
$$

Let us apply the definition of the stability. When

$$
\left\|\left(\begin{array}{c}
x_{1}(0) \\
x_{2}(0)
\end{array}\right)-\left(\begin{array}{c}
\bar{x}_{1}(0) \\
\bar{x}_{2}(0)
\end{array}\right)\right\|=\left\|\left(\begin{array}{l}
c_{1} \\
c_{2}
\end{array}\right)-\left(\begin{array}{l}
0 \\
0
\end{array}\right)\right\| \leq\left|c_{1}\right|+\left|c_{2}\right|<\delta,
$$

then

$$
\begin{gathered}
\left\|\left(\begin{array}{l}
x_{1}(t) \\
x_{2}(t)
\end{array}\right)-\left(\begin{array}{l}
0 \\
0
\end{array}\right)\right\|=\left\|\left(\begin{array}{c}
c_{1} \cos t+c_{2} \sin t \\
-c_{1} \sin t+c_{2} \cos t
\end{array}\right)-\left(\begin{array}{l}
0 \\
0
\end{array}\right)\right\| \\
\leq 2\left|c_{1}\right|+2\left|c_{2}\right| \\
<2 \delta=\varepsilon, \quad \delta=\varepsilon / 2 .
\end{gathered}
$$

This means that the zero solutions of the equation is uniformly stable.

Definition 2.11. The solution $x(t)$ of EQ.(4) is called asymptotically stable if it is stable and if there exists a $\delta_{0}>0$ such that $\left\|\bar{x}_{0}-x(t)\right\| \leq \delta_{0}$ implies $\|\bar{x}(t)-x(t)\| \rightarrow 0$ as $t \rightarrow \infty$.

Consider the above first example. When we take into consideration it solution

$$
\begin{gathered}
x_{1}(t)=c_{1} e^{-t}+c_{2} e^{-2 t}, \quad t \geq 0 \\
x_{2}(t)=-c_{1} e^{-t}-2 c_{2} e^{-2 t},
\end{gathered}
$$

clearly,

$$
x_{1}(t) \rightarrow 0, \quad x_{2}(t) \rightarrow 0 \text { as } t \rightarrow \infty, .
$$

Then the zero solution is also asymptotically stable.

Definition 2.12. The solution $x(t)$ of EQ.(4) is known as unstable if it is not stable.

Example 2.13. Consider the initial value problem

$$
x^{\prime \prime}-3^{\prime}+2 x=0, \quad x(0)=1, \quad x^{\prime}(0)=3 .
$$

Let $x=x_{1}, \quad x_{1}^{\prime}=x_{2}$. Hence, we have

$$
x_{1}^{\prime}=x_{2}, \quad x_{2}^{\prime}=-2 x_{1}+3 x_{2} .
$$

It follows that

$$
\begin{gathered}
x_{1}(t)=c_{1} e^{t}+c_{2} e^{2 t}, \quad t \geq 0 \\
x_{2}(t)=c_{1} e^{t}+2 c_{2} e^{2 t} .
\end{gathered}
$$

When we use the above initial conditions, we get

$$
x_{1}(t)=-e^{t}+2 e^{2 t}
$$




$$
x_{2}(t)=-e^{t}+4 e^{2 t} .
$$

Let us apply the definition of the stability. When

$$
\left\|\left(\begin{array}{c}
x_{1}(0) \\
x_{2}(0)
\end{array}\right)-\left(\begin{array}{c}
\bar{x}_{1}(0) \\
\bar{x}_{2}(0)
\end{array}\right)\right\|=\left\|\left(\begin{array}{c}
1 \\
-3
\end{array}\right)-\left(\begin{array}{l}
0 \\
0
\end{array}\right)\right\|<4<\delta
$$

then it follows that

$$
\begin{gathered}
\left\|\left(\begin{array}{c}
x_{1}(t) \\
x_{2}(t)
\end{array}\right)-\left(\begin{array}{l}
0 \\
0
\end{array}\right)\right\|=\left\|\left(\begin{array}{c}
-e^{t}+2 e^{2 t} \\
-e^{t}+4 e^{2 t}
\end{array}\right)-\left(\begin{array}{l}
0 \\
0
\end{array}\right)\right\| \\
\leq\left|-e^{t}\right|+\left|2 e^{2 t}\right|+\left|-e^{t}\right|+\left|4 e^{2 t}\right| \not \leq \varepsilon,
\end{gathered}
$$

when $t$ sufficiently bigger.

This means that the zero solution of the system is unstable.

Definition 2.14. The solution $x(t)$ of EQ.(4) is called uniformly asymptotically stable if it is uniformly stable and there is a $\delta_{0}>0$ and $, \forall \eta>0, \exists T=T(\eta)>0$ S.T the inequalities $t_{1} \geq t_{0}$ and $\left\|\bar{x}\left(t_{1}\right)-x\left(t_{1}\right)\right\| \leq$ $\delta_{0}$ imply $\|\bar{x}(t)-x(t)\|<\eta$ for all $t \geq t_{1}+T$.

Definition 2.15.(Lyapunov Function) is any differentiable and continuous function $V: G \rightarrow R$, such that $V(t, x)$ are positive defined $\forall t \in\left[t_{0},+\infty\right), x \in R^{n}$, also the time derivative of this function is quasi-specific negative, given negative or positive definite.

Consider the general non - autonomous delay differential system:

$$
x^{\prime}=F\left(t, x_{t}\right) \cdot x_{t}=x(t+\theta), \quad-r \leq \theta<0, \quad t \geq 0,
$$

Where $F:[0, \infty) \times C_{H \rightarrow} \mathcal{R}^{n}$ is a continuous mapping, $F(t, 0)=0$, and we assume that $\mathrm{F}$ takes closed bunded sets into bunded set of $\mathcal{R}^{n}$ here $(C,\|\|$.$) is the Bunch space of continuous function \phi:[-r, 0] \rightarrow$ $\mathcal{R}^{n}$ with supremam norm; $r>0, C_{H}$ is open ball in $C, C_{H}=\left\{\phi \in\left([-r, 0], \mathcal{R}^{n}\right):\|\phi\|<H\right\}$.

Theorem 2.16. (Burton [2]). Assume that a Lyapunov function for EQ.(4) and wedges satisfying;

(i) $W_{1}(|\varphi(0)|) \leq V(t, \varphi) \leq W_{2}(\|\varphi\|)$, (where $W_{1}(r)$ and $W_{2}(r)$ are wedges),

(ii) $V^{\prime}(t, \varphi) \leq 0$

Then the zero solution of EQ.(4) is uniformly stable.

Theorem 2.17. (Yoshizawa [37]). Suppose that there exists a continuous Lyapunov function $V(t, \varphi)$ defined for all $t \in \mathcal{R}^{+}$and $\varphi \in S$, which satisfies the following conditions;

(i) $a(|\varphi(0)|) \leq V(t, \varphi) \leq b_{1}(|\varphi(0)|)+b_{2}(\|\varphi\|)$,

Where $a(r), b_{1}(r), b_{2}(r) \in C I,(C I)$ denotes the families of continuous increasing functions), and are positive for $r>H$ and $a(r)-b_{2}(r) \rightarrow \infty$ as $r \rightarrow \infty$.

(ii) $V^{\prime}(t, \varphi) \leq 0$.

Then, the solutions of EQ.(4) are uniformly bounded.

Theorem 2.18. If there is a Lyapunov function for EQ.(4) and wedges S.T

$$
W_{1}\left(|(0)| \leq V(t,) \leq W_{2}(\|\|),\right.
$$

and

$$
V_{(1)}^{\prime}\left(t, x_{t}\right) \leq-W_{3}(|x(t)|) .
$$

Then $x=0$ is uniformly asymptotical stable.

\section{Application of the Lyapunov's second method}

3.1. The Lyapunov's first method. By solving an initial value problem, after finding solutions, we apply definition of the stability whether the founded solution is stable or not. This method is called the Lyapunov's first method. 
3.2. The Lyapunov's second (direct) method. Without solving any initial value problem, by define a suitable Lyapunuov function, we can determine the stability of solution subject to the Lyapunov's theorems; this method is called Lyapunov's second method.

We begin with the scalar equation

$$
x^{\prime}(t)=-a x(t)+b x(t-r)
$$

where $\mathrm{a}, \mathrm{b}$, and $\mathrm{r}$ are constants, $\mathrm{r} \geq 0$.

Theorem3.3. If $a>0$ and $|b|<a$, then the zero solution for EQ.(5) above is uniformaly asymptoticaly stable for every $r \geq 0$.

Proof. Define Lyapunov functional

$$
V(t)=x^{2}(t)+|b| \int_{t-r}^{t} x^{2}(s) d s .
$$

The derivative of $\mathrm{V}$ along an unknown solution of Eq.(5) satisfies

$$
\begin{aligned}
& V^{\prime}(t)=2 x[-a x+b x(t-r)]+|b| x^{2}-|b| x^{2}(t-r) \\
& \quad \leq-2 a x^{2}+|b| x^{2}+|b| x^{2}(t-r)+|b| x^{2}-|b| x^{2}(t-r) \\
& \quad=[-2 a+2|b|] x^{2} \\
& =-\alpha x^{2}
\end{aligned}
$$

where $\alpha=2 a-2|b|>0$. We have

$$
|x(t)|^{2} \leq V(t) \leq(1+|b| r)\left\|x_{t}\right\|^{2},
$$

and

$$
V^{\prime}(t) \leq-2[a-|b|]|x|^{2}=\alpha x^{2}
$$

In view of Theorems (2.16) and (3.3), we can conclude the zero solution of EQ.(5) is stable, uniformly stable, uniformly asymptotical stable, and al solutions are uniformly bounded, too. It should be noted that without solving EQ.(5), we can determine the stability, uniform stability, uniformly roundedness and uniformly asymptotical stability of solutions of equation considered. This shows the advantage of the Lyapunov's second method.

We are now discussing the stability of the same equation with the fixed point theory:

Proof. Let $\psi:[-\mathrm{r}, 0] \rightarrow \mathrm{R}$ be continuous initial function and let $(\mathrm{M},\|\cdot\|)$ be the complete metric space of continuous functions $\phi:[-\mathrm{r}, \infty) \rightarrow \mathrm{R}$ with $\phi_{0}=\psi$ and $\psi(\mathrm{t}) \rightarrow 0$ as $\mathrm{t} \rightarrow \infty$. Recall that the notation $\phi_{0}=\psi$ means that $\phi(t)=\psi(t)$ for $-\mathrm{r} \leq \mathrm{t} \leq 0$. Use the variation of parameters formula is

$$
x(t)=\psi(0) e^{-a t}+\int_{0}^{t} e^{-a(t-s)} b x(s-r) d s,
$$

and use this to define a mapping $\mathrm{P}: \mathrm{M} \rightarrow \mathrm{M}$ by $\phi \in \mathrm{M}$ and $-\mathrm{r} \leq \mathrm{t} \leq 0$ implies that

$$
(P \phi)(t)=\psi(0) e^{-a t}+\int_{0}^{t} e^{-a(t-s)} b \phi(s-r) d s .
$$

It is clear that $\phi \in \mathrm{M}$. To prove that $\mathrm{P}$ is a contraction, suppose that $\phi, \eta \in \mathrm{M}$. Then

$$
\begin{gathered}
|(P \phi)(t)-(P \eta)(t)| \leq \int_{0}^{t}|b| e^{-a(t-s)}|\phi(s-r)-\eta(s-r)| d s \leq\|\phi-\eta\| \int_{0}^{t}|b| e^{-a(t-s)} d s \\
\leq\|\phi-\eta\|\left(\frac{|b|}{a}\right) .
\end{gathered}
$$


This is a contraction since $\mathrm{a}>|\mathrm{b}|$ and so $\mathrm{P}$ had the unique fixed point $\phi \in \mathrm{M}$. Since the fixed point $\phi$ resides in $\mathrm{M}$ it follows that $\phi(\mathrm{t}) \rightarrow 0$. Of course from EQ.(6), we see that the fixed point solves EQ.(5). Moreover, to prove stability, we apply norms to both sides of EQ.(6) at the fixed point and obtain

$$
\|\phi\| \leq\|\psi\|+\left(\frac{|b|}{a}\right)\|\phi\| .
$$

From which the stability relation is easily derived.

We motivate our next example by considering the following delay differential equation with variable coefficients:

$$
x^{\prime}(t)=-a(t) x(t)-b(t) x(t-r),
$$

where $\mathrm{a}$ and $\mathrm{b}$ are bounded continuous functions,

$$
a(t) \geq \delta>0, \quad|b(t)| \leq \theta \delta, \quad \theta<1 .
$$

It should be noted that this delay differential equation includes the preceding delay differential equation. Using the Lyapunov functional

$$
V\left(t, x_{t}\right)=\left(\frac{1}{2}\right) x^{2}(t)+\left(\frac{1}{2}\right) \delta \int_{t-r}^{t} x^{2}(s) d s
$$

With the triangle inequality, we have

$$
\mathrm{V}^{\prime} \leq\left(\frac{\delta}{2}\right)(\theta-1)\left(\mathrm{x}^{2}(\mathrm{t})+\mathrm{x}^{2}(\mathrm{t}-\mathrm{r})\right)
$$

It can now be argued that this yields uniformly asymptotically stability of solutions.

Remark3.4. Consider the delay differential equation of the form

$$
x^{\prime}(t)=-a x(t)+b x(t-r) .
$$

It follows that the assumptions $a>0$ and $|b|<a$ guarantee, then the stability of zero solution given EQ.(5). Next, when we apply the fixed point theory it follows that the assumption $a>|b|$ guarantee stability of zero solution of above equation, too. From which it follows that when we apply the Lyapunov second method and the fixed point theory, then the assumptions of theorems are the same for delay differential equation of first order with the constant coefficients.

Consider the differential equation of first order linear delay with variable delay of the model

$$
x^{\prime}(t)=-a(t) x(t)+b(t) x(t-g(t)),
$$

Theorem3.5. We assume the following condition satisfied:

$g^{\prime}(t)$ is bounded and $1-g^{\prime}(t)>0,-2 \mathrm{a}(\mathrm{t})+1 \leq-\left(1-\mathrm{g}^{\prime}(\mathrm{t})\right)$, and $|b(t)| \leq \alpha\left(1-g^{\prime}(t)\right)$, for some $\alpha \in(0,1)$. So the zero solution of EQ.(7) is uniformaly asymptoticaly stable.

Proof. Define Lyapunov functional $V(t)$ by

$$
V(x)=x^{2}+\int_{t-g(t)}^{t} x^{2}(s) d s
$$

It's clear

$V(0)=0$, and $V(x) \geq x^{2}$.

This indicates that $V(x)$ is positive. Then, along with solutions EQ.(7), we have

$$
V^{\prime}(x)=2 x(t)(-a(t) x(t)+b(t) x(t-g(t)))
$$




$$
\begin{gathered}
+x 2(t)-\left(1-g^{\prime}(t)\right) x^{2}(t-g(t)) \\
\leq-2 a(t) x^{2}(t)+|b(t)| x^{2}(t)+|b(t)| x^{2}(t-g(t)) \\
+x 2(t)-\left(1-g^{\prime}(t)\right) x^{2}(t-g(t)) \\
=(-2 a(t)+|b(t)|+1) x^{2}(t) \\
+\left(|b(t)|-\left(1-g^{\prime}(t)\right)\right) x^{2}(t-g(t)) \\
\leq(\alpha-1)\left(1-g^{\prime}(t)\right)\left(x^{2}(t)+x^{2}(t-g(t))\right) \\
\leq(\alpha-1)\left(1-g^{\prime}(t)\right) x^{2}(t) .
\end{gathered}
$$

In view of the conditions $0<\alpha<1$ and $1-g^{\prime}(t)>0$, it follows that $V^{\prime}(x)$ is negative defined. Through theory 10, we deduce that the zero solution of EQ.(7) is uniformly asymptotically stable.

Now we will prove the stability result for EQ.(7) by fixed point theory

Theorem3.6. We supposes that the following assumptions contract: $a, b$, and $g$ are continuous functions such that

$$
\int_{0}^{t} a(s) d s \rightarrow \infty \text { as } t \rightarrow \infty,
$$

there is an $\alpha<1$ with

$$
\int_{0}^{t} e^{-\int_{s}^{t} a(u) d u}|b(s)| d s \leq \alpha, \quad t \geq 0,
$$

$0 \leq r(t), \quad t-r(t) \rightarrow \infty$ as $t \rightarrow \infty$, there is an $L>0,|x|,|y| \leq L, g(0)=0$ and $|g(x)-g(y)| \leq$ $|x-y|$. Then, every solution of EQ. (7) with small continuous initial function tends to o as $t \rightarrow \infty$. So the zero solution is stable.

Proof. We will take $\mathrm{t}_{0}=0$. For the $\alpha$ and $\mathrm{L}$, find $\delta>0$ with $\delta+\alpha L \leq L$. Let $\psi:(-\infty, 0] \rightarrow R$ be a given continuous function with $|\psi(t)|<\delta$ \& let

$S=\{\phi: R \rightarrow R\|\phi\| \leq L, \phi(t)=\psi(t)$ if $\mathrm{t} \leq 0, \phi(t) \rightarrow 0$ as $\mathrm{t} \rightarrow \infty, \phi \in C\}$, where $\|$.$\| is the supremum metric. Then (S,\|\cdot\|)$ is complete metric space.

Define $P: S \rightarrow S$ by $(P \phi)(t)=\psi(t)$ if $t \leq o$ and for $t \geq o$ let

$$
(P \phi)(t)=e^{-\int_{0}^{t} a(s) d s} \psi(0)+\int_{0}^{t} e^{-\int_{s}^{t} a(u) d u} b(s) x(s-g(s)) d s .
$$

Clearly, $P \phi \in C$. We now show that $(P \phi)(t) \rightarrow 0$ as $t \rightarrow \infty$. Let $\phi \in S$ and $\varepsilon>0$ be given. Then $\|\phi\| \leq L$, there exists $t_{1}>0$ with $\left.\mid ?(t)-g(t)\right) \mid<\varepsilon$ if $t \geq t_{1}$, and there exists $t_{2}>t_{1}$ such that $t>t_{2}$ implies that $e^{-\int_{t_{1}}^{t} a(u) d u}<\frac{\varepsilon}{L \alpha}$. Thus, $t>t_{2}$ implies that

$$
\begin{gathered}
\left|\int_{0}^{t} e^{-\int_{s}^{t} a(u) d u} b(s) \phi(s-g(s)) d s\right| \\
\leq \int_{0}^{t_{1}} e^{-\int_{s}^{t} a(u) d u}|b(s)| L d s+\int_{t_{1}}^{t} e^{-\int_{s}^{t} a(u) d u}|b(s)| \varepsilon d s \\
\leq e^{-\int_{t_{1}}^{t} a(u) d u} \int_{0}^{t_{1}} e^{-\int_{s}^{t_{1}} a(u) d u}|b(s)| L d s+\alpha \varepsilon
\end{gathered}
$$




$$
\leq \alpha L e^{-\int_{t_{1}}^{t} a(u) d u}+\alpha \varepsilon \leq \varepsilon+\alpha \varepsilon .
$$

To see that $\mathrm{P}$ is contraction under the supremum metric, if $\phi, \eta \in S$, then

$$
\begin{aligned}
|(P \phi)(t)-(P \eta)(t)| & =\left|\int_{0}^{t} e^{-\int_{s}^{t} a(u) d u} b(s)(\phi(s-r)-\eta(s-r)) d s\right| \\
& \leq \int_{0}^{t} e^{-\int_{s}^{t} a(u) d u}|b(s)|\|\phi-\eta\| d s \leq \alpha\|\phi-\eta\| .
\end{aligned}
$$

With $\alpha<1$ by the condition $\int_{0}^{t} e^{-\int_{s}^{t} a(u) d u}|b(s)| d s \leq \alpha, \quad t \geq 0$, Hence, $\mathrm{P}$ has a unique fixed point in $\mathrm{S}$ which shows that every solutions EQ.(7) tends to zero, and moreover the zero solution of EQ.(7) is stable.

Now we study the stability and asymptotically stability of the zero solution of EQ.(7) by funding the Lyapunov's direct method.

Remark3.7. Consider the linear delay differential equation of first order with variable delay of the model

$$
x^{\prime}(t)=-a(t) x(t)+b(t) x(t-g(t)) .
$$

It follows that, when we apply the Lyapunov's direct method, the following assumptions $g^{\prime}(t)$ is bounded,

$$
1-g^{\prime}(t)>0,-2 a(t)+1 \leq-\left(1-g^{\prime}(t)\right),
$$

and $|b(t)| \leq \alpha\left(1-g^{\prime}(t)\right)$, for some $\alpha \in(0,1)$.

Are suficient for the uniformly asymptotically stability of zero solution of EQ.(7). Besides, when we apply fixed point theory, instate of the mentioned conditions, the following assumptions guarantee the asymptotically stability of zero solution:

$$
\begin{gathered}
\int_{0}^{\mathrm{t}} \mathrm{a}(\mathrm{s}) \mathrm{ds} \rightarrow \infty \text { as } \mathrm{t} \rightarrow \infty, \\
\int_{0}^{t} e^{-\int_{s}^{t} a(u) d u}|b(s)| d s \leq \alpha, \alpha<1 \quad t \geq 0, \\
\mathrm{o} \leq \mathrm{r}(\mathrm{t}), \quad \mathrm{t}-\mathrm{r}(\mathrm{t}) \rightarrow \infty \text { as } \mathrm{t} \rightarrow \infty,
\end{gathered}
$$

and there is an $L>0$ so that if $|x|,|y| \leq L$ then $g(0)=0$ and

$$
|g(x)-g(y)| \leq|x-y|
$$

In the application of the Lyapunov method, the differentiability of the function $g$ is needed, however, this condition is not needed for fixed point theories. This is a disadvantage for the Lyapunov method, however, an advantage in the case of applications of fixed point theories. However, the case of application of fixed point theories, we want impose the condition.

$$
\int_{0}^{t} e^{-\int_{s}^{t} a(u) d u}|b(s)| d s \leq \alpha, \quad 0<\alpha<1, \quad t \geq 0 .
$$

This is a stronger condition. However, during the application of Lyapunov method, we don't need this condition. This is advantage of Lyapunov method.

Construction or definition of a Lyapunov functional is needed when we apply this method. This is the disadvantage of this method. We can give more discussion about the application of these methods. But, we would not like to give here more details.

We now consider non-autonomous differential equation of second order

$$
x^{\prime \prime}(t)+f\left(t, x(t), x^{\prime}(t)\right) x^{\prime}(t)+b(t) g(x(t-\tau(t)))=q(t)(8)
$$


And also we can write out the EQ.(8) in the sample order as follows $x^{\prime}(t)=y(t)$

$$
y^{\prime}(t)=-f(t, x(t), y(t)) y(t)-b(t) g(x(t))+b(t) \int_{t-\tau(t)}^{t} g^{\prime}(x(s)) y(s) d s+q(t),
$$

Where deviation $\tau(t)$ is the median of the variable deviation, $f, b, q$ and $g$ are continuous functions in $\mathcal{R}^{3}, \mathcal{R}$ and $[0, \infty)$ respectively, and are also dependent on arguments candor presented with $g(0)=0$. Continoity these functions are suficient requirements to equate the solution of EQ.(8). It is also assumed that the functions $\mathrm{f}$ and $g$ meet the Lipchitz state in numbers $x, x^{\prime}$ and $x(t-\tau(t))$. Through this assumption the uniqueness of EQ. (8) solutions is guaranteed. The derivatives $\left(\frac{d g}{d x}=g^{\prime}(x)\right)$ is occur and continuous.

By determining the Lyapunov function (se Krasonvskii [23]) sufficient conditions for stabilization and limits of solutions Eq.(8) are obtained.

We prove the following theorem by the Lyapunov's second method.

Theorem 3.8. Further assuming the basic concepts for the functions $\mathrm{f}, \mathrm{b}$ and $\mathrm{g}$ we assume that the continuum has a constant $\mathrm{L}$, hence to keep the following terms:

$t-\tau(t)$ is strictly increasing, $\lim _{t \rightarrow \infty}(t-\tau(t))=\infty$ and $b(t)$ is decreasing,

$$
\begin{gathered}
1-\tau^{\prime}(t)-b(t) \geq 0, f(t, x, y) \geq a(t) \geq 2^{-1} L(b(t)+1) \tau(t)=0, \\
\left|g^{\prime}(x)\right|=L .(x \neq 0), x g(x)>0, g(0)=0,
\end{gathered}
$$

Hence the zeroes solution of EQ.(4) is stable.

Proof. Defining the Lyapunov function

$$
V(t)=\exp \left(-2 \int_{0}^{t}|e(s)| d s\right)\left\{b(t) \int_{0}^{x} g(s) d s+\frac{1}{2} y^{2}+1+\lambda \int_{-\tau(t)}^{0} \int_{t+s}^{t} y^{2}(\theta) d \theta d s\right\},
$$

Where $\lambda>0$, which will be obtained later and $e(t)$ is a continuous function on $\mathcal{R}^{+}=[0, \infty)$ and $\int_{0}^{t}|e(s)| d s<\infty$, then it follows that

$$
\frac{1}{2} y^{2}+1+\lambda \int_{-\tau(t)}^{0} \int_{t+s}^{t} y^{2}(\theta) d \theta d s \leq V(t) b(t) \int_{0}^{x} g(s) d s \exp ?\left(-2 \int_{0}^{\infty}|e(s)| d s\right)
$$

By the time derived the Lyapunov function $V(t)$ over the system (5), we have

$$
\begin{gathered}
=2|e(t)| \exp ?\left(-2 \int_{0}^{t}|e(s)| d s\right)\left\{b(t) \int_{0}^{x} g(s) d s+\frac{1}{2} y^{2}+1+\lambda \int_{-\tau(t)}^{0} \int_{t+s}^{t} y^{2}(\theta) d \theta d s\right\} \dot{V}(t) \\
b^{\prime}(t) \int_{0}^{x} g(s) d s+\exp \left(-2 \int_{0}^{t}|e(s)| d s\right)\{f(t, x, y)-\lambda \tau(t)\} y^{2}-\exp \left(-2 \int_{0}^{t}|e(s)| d s\right) \\
\left\{y b(t) \int_{t-\tau(t)}^{t} g^{\prime}(x(s)) y(s) d s+\exp \left(-2 \int_{0}^{t}|e(s)| d s\right)-\lambda\left\{1-\tau^{\prime}(t)\right\} \int_{t-\tau(t)}^{t} y^{2}(s) d s\right\} .
\end{gathered}
$$

By assumptions of Theorem (3.1) and the estimate $|\mathrm{mn}| \leq \frac{\mathrm{m}^{2}}{2}+\frac{\mathrm{n}^{2}}{2}$, it follows that

$$
\left\{y b(t) \int_{t-\tau(t)}^{t} g^{\prime}(x(s)) y(s) d s \leq \frac{L b(t) \tau(t)}{2} y^{2}+\frac{L b(t)}{2} \int_{t-\tau(t)}^{t} y^{2}(s) d s\right\} .
$$

Hence

$$
\begin{aligned}
& \exp \left(-2 \int_{0}^{t}|e(s)| d s\right) y^{2}-\left\{a(t)-\lambda\{\tau(t)\}-2^{-1} L b(t) \tau(t) y^{2} \leq \dot{V}(t)\right. \\
= & \left\{\left[2^{-1} L b(t)-\lambda\left(1-\tau^{\prime}(t)\right)\right] \tau \int_{t-\tau(t)}^{0} y^{2}(s) d s\right\} .+\exp \left(-2 \int_{0}^{t}|e(s)| d s\right) .
\end{aligned}
$$


Let $\lambda=\frac{L}{2}$, so that

$$
\begin{gathered}
\exp \left(-2 \int_{0}^{t}|e(s)| d s\right) y^{2}-\left\{a(t)-2^{-1} L(b(t)+1) \tau(t) \leq \dot{V}(t)\right. \\
\left.2^{-1} L\left\{1-\tau^{\prime}(t)-b(t)\right\} \exp \left(-2 \int_{0}^{t}|e(s)| d s\right) \int_{t-\tau(t)}^{0} y^{2}(s) d s\right\} \leq 0,
\end{gathered}
$$

by assumptions of Theorem (3.6). Hence, the zeroes solution of EQ.(8) is stable by Theorem (2.16).

Corollary3.9. If Theorem.(3.6) Assumptions are assumed, and then by Theorem (2.17), we deduce that the zero solution for the equation (8) uniform stable.

Let $q(t) \neq 0$.

We now discuss the stability of the same equation by fixed point theory.

We can write Eq.(15), when $q(t)=0$ as $x^{\prime}=y$,

$$
y^{\prime}=-f(t, x, y) y-b(t) g(x(t-\tau(t))) .
$$

For each $t_{0} \geq 0$, definem $\left(t_{0}\right)=\inf \{s-\tau(s): s \geq 0\}$. Let $C\left(t_{0}\right)=C\left(\left[m\left(t_{0}\right), t_{0}\right], R\right)$, With standard continuous function $\|$.$\| , where \|\psi(s)\|=\left\{\sup |\psi(s)|: m\left(t_{0}\right) \leq s \leq t_{0}\right\}$. It Will not cause any confusion even if we using $\|\psi(s)\|$ us supremam on $\left[m\left(t_{0}\right), \infty\right)$. It known in [2], for a given continuous function $\Phi$ and for the number $y_{0}$, there is a solution for the system (10) at an interval $\left[t_{0}, T\right)$ if the solution residue selected, then $T=\infty)$.

Let $A(t)=f(t, x(t), y(t)$. So the system (10) can write as

$$
\begin{array}{r}
\mathrm{x}=\mathrm{y}, \\
y^{\prime}=-A(t) y-b(t) g(x(t-\tau(t))) .
\end{array}
$$

$\mathrm{Pi}[19]$ proved the followings theory through fixed points.

Theorem3.10. We suppose that the following assumptions hold:

(i) $t-\tau(t)$ is increasing, $\lim _{t \rightarrow \infty} m t-\tau(t)=\infty$. The inverse of $t-\tau(t)$ exists, denoted by $p(t)$.

$0 \leq b(t) \leq M$ for $M \in R$.

(ii) $\exists l>0$ such that $g(x)$ satisfies the Lipchitz condition on $[-l, l] . g(x)$ is odd and it is increasing on $[-l, l], x-g(x)$ is no decreasing on $[0, l]$.

(iii) $\exists \alpha \in(0,1)$ and a continuous function $a(t):[0, \infty) \rightarrow[0, \infty)$ S.T $f(t, x, y) \geq a(t)$ for $t \geq 0, x, y \in R$

$$
\sup _{t \geq 0} \int_{t}^{P(t)} \int_{0}^{\infty} e^{-\int_{s}^{\omega+s} a(v) d v} b(s) d \omega d s+2 \sup _{t \geq 0} \int_{0}^{t} \int_{t-s}^{\infty} e^{-\int_{s}^{\omega+s} a(v) d v} b(s) d \omega d s \leq \alpha .
$$

(iv) $\exists a_{0}>0$ and $Q>0$ such that for each $t \geq 0$, if $J \geq Q$, then

$$
\begin{gathered}
\int_{t}^{t+J} a(v) d v \geq a_{0} J . \\
\left(\left[\mathrm{t}_{0}-\tau, \mathrm{t}_{0}\right], \mathcal{R}\right),\|\psi(\mathrm{s})\|=\left\{\sup |\psi(\mathrm{s})|: \mathrm{m}\left(\mathrm{t}_{0}\right) \leq \mathrm{s} \leq \mathrm{t}_{0}\right\} .
\end{gathered}
$$

Then $\exists d \psi(0, l)$ such that for each $\psi:\left[m\left(t_{0}\right), t_{0}\right] \rightarrow \mathfrak{R}$ initial function and $x^{\prime}\left(t_{0}\right)$ satisfying $\left|x^{\prime}\left(t_{-} 0\right)\right|+$ $\|\psi(s)\| \leq \delta$, there is a unique continuous function $x:\left[m\left(t_{0}\right), \infty\right] \rightarrow \mathfrak{R}$ satisfies $x(t)=\psi(t), \quad t \in$ $\left[m\left(t_{0}\right), t_{0}\right]$, which is solution of EQ.(8) at $\left[t_{0}, \infty\right]$. Furthermore, the zero solution of EQ.(8) is stable (Pi [19]).

We produce the following basic assumptions about the delay function $t(t)$ in EQ.(8):

$(\mathcal{A}) t-\tau(t)$ is increasing, $\lim _{t \rightarrow \infty} t-\tau(t)=\infty$. The inverse of $t-\tau(t)$ exist, denoted by $p(t), 0 \leq b(t) \leq$ $M$, for some constant $M>0$. 
Lemma3.11. Let $\psi:\left[m\left(t_{0}\right), t_{0}\right] \rightarrow R$ be a given continuous function. If $x(t), y(t)$ is the solution of the system (10) on $\left[t_{0}, T_{1}\right]$ satisfying $x(t)=\psi(t), t \in\left[m\left(t_{0}\right), t_{0}\right]$, and $y\left(t_{0}\right)=x^{\prime}\left(t_{0}\right)$, then $x(t)$ is solution of integral equation

$$
\begin{aligned}
& x(t)=\psi\left(t_{0}\right) e^{-\int_{t_{0}}^{t} \hat{D}(s) d s}+\int_{t_{0}}^{t} e^{-\int_{u}^{t} \hat{D}(s) d s} B(u) d u
\end{aligned}
$$

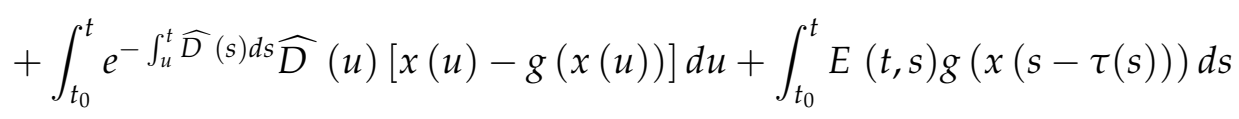

$$
\begin{aligned}
& +\int_{t-\tau(t)}^{t} \widehat{D}(s) g(x(s)) d s-e^{-\int_{u}^{t} \hat{D}(s) d s} \times \int_{t_{0}-\tau\left(t_{0}\right)}^{t} \widehat{D}(s) g(x(s)) d s \\
& -\int_{t_{0}}^{t}\left[\int_{u-\tau(u)}^{t} \hat{D}(s) g(x(s)) d s\right] e^{-\int_{u}^{t} \hat{D}(s) d s} \hat{D}(u) d u-\int_{t_{0}}^{t} e^{-\int_{u}^{t} \hat{D}(s) d s} \\
& {\left[\int_{t_{0}}^{u} E(u, s) g(x(s-\tau(s))) d s\right] \widehat{D}(u) d u \text {. }}
\end{aligned}
$$

Conversely, if the continuous function $x(t)=\psi(t), t \in\left[m\left(t_{0}\right), t_{0}\right]$, is the solution of integral Eq. (12) on $\left[t_{0}, T_{2}\right]$, then $(x(t), y(t))$ is a solution of system (10) on $\left[\mathrm{t}_{0}, \mathrm{~T}_{2}\right]$.

Proof. Let $f_{1}(t, x(t), y(t))=A(t)$.

Then, system (10), can be stated as follows

$$
\begin{gathered}
x^{\prime}=y, \\
y^{\prime}=-A(t) y-b(t) g(x(t-\tau(t)))
\end{gathered}
$$

So that

$$
y^{\prime}+A(t) y+b(t) g(x(t-\tau(t)))=0
$$

Multiplying both sides of Eq.(13) by $e^{-\int_{\infty}^{t} A(s) d s}$ and integrating, it follows that

$$
y(t)=y\left(t_{0}\right) e^{-\int_{t_{0}}^{t} A(s) d s}-\int_{t_{0}}^{t} e^{-\int_{u}^{t} A(s) d s} b(u) g(x(u-\tau(u))) d u
$$

so that

$$
x^{\prime}(t)=y(t)=x^{\prime}\left(t_{0}\right) e^{-\int_{t_{0}}^{t} A(s) d s}-\int_{t_{0}}^{t} e^{-\int_{u}^{t} A(s) d s} b(u) g(x(u-\tau(u))) d u .
$$

If we choose $x^{\prime}\left(t_{0}\right) e^{-\int_{t_{0}}^{t} A(s) d s}=B(t)$, then from Eq.(14) we have

Let

$$
\begin{gathered}
e^{-\int_{u}^{t} A(s) d s} b(u)=C(t, u), \\
\int_{t_{0}}^{\infty} C\left(u+t-t_{0}\right) d u=D(t) \geq 0, \\
\frac{D(t)}{1-\tau(t)}=\tilde{D}(t), \\
\tilde{D}(p(t))=\hat{D}(t),
\end{gathered}
$$

and

$$
\int_{t_{0}+t-s}^{\infty} C\left(u+s-t_{0}, s\right) d u=E(t, s) \geq 0
$$

Hence, it follows

$$
x^{\prime}(t)=B(t)-g(x(t-\tau(t))) \int_{t_{0}}^{\infty} C\left(u+t-t_{0}, t\right) d u+\frac{d}{d t} \int_{t_{0}}^{t} E(t, s) g(x(s-\tau(s))) d s
$$


so that

$$
\begin{gathered}
x^{\prime}(t)=B(t)-g(x(t-\tau(t))) D(t)+\frac{d}{d t} \int_{t_{0}}^{t} E(t, s) g(x(s-\tau(s))) d s \\
=B(t)-\tilde{D}(P(t)) g(x(t))+\frac{d}{d t} \int_{t-\tau(t)}^{t} \widetilde{D}(P(s)) g(x(s)) d s+\frac{d}{d t} \int_{t_{0}}^{t} E(t, s) g(x(s-\tau(s))) d s .
\end{gathered}
$$

Then

$$
\begin{gathered}
x^{\prime}(t)+\hat{D}(t) x(t)=B(t)+\hat{D}(t)[x(t)-g(x(t))]+\frac{d}{d t} \int_{t-\tau(t)}^{t} \hat{D}(s) g(x(s)) d s \\
+\frac{d}{d t} \int_{t_{0}}^{t} E(t, s) g(x(s-\tau(s))) d s .
\end{gathered}
$$

Multiply both sides of Eq.(15) by $e^{-\int_{t_{0}}^{t} \hat{D}(s) d s}$ and then integrate for all $t \in\left[t_{0}, T_{1}\right]$, we have

$$
\begin{gathered}
x(t)=?\left(t_{0}\right) e^{-\int_{t_{0}}^{t} \hat{D}(s) d s}+\int_{t_{0}}^{t} e^{-\int_{u}^{t} \hat{D}(s) d s} B(u) d u \\
+\int_{t_{0}}^{t} e^{-\int_{u}^{t} \hat{D}(s) d s} \hat{D}(t)[x(u)-g(x(u))] d u \\
+\int_{t_{0}}^{t} e^{-\int_{u}^{t} \hat{D}(s) d s}\left[\frac{d}{d u} \int_{u-\tau(u)}^{u} \hat{D}(s) g(x(s)) d s\right] d u \\
+\int_{t_{0}}^{t} e^{-\int_{u}^{t} \hat{D}(s) d s}\left[\frac{d}{d u} \int_{t_{0}}^{u} E(u, s) g(x(s-\tau(s))) d s\right] d u
\end{gathered}
$$

The right hand side of the above estimated, by integrating the 4 th, 6 th $\& 7$ th terms, it follows

$$
\begin{gathered}
x(t)=?\left(t_{0}\right) e^{-\int_{t_{0}}^{t} \hat{D}(s) d s}+\int_{t_{0}}^{t} e^{-\int_{u}^{t} \hat{D}(s) d s} B(u) d u \\
+\int_{t_{0}}^{t} e^{-\int_{u}^{t} \hat{D}(s) d s} \hat{D}(u)[x(u)-g(x(u))] d u \\
+\int_{t_{0}}^{u} E(t, s) g(x(s-\tau(s))) d s+\int_{t-\tau(t)}^{u} \hat{D}(s) g(x(s)) d s-e^{-\int_{t_{0}}^{t} \hat{D}(s) d s} \\
\times \int_{t_{0}-\tau\left(t_{0}\right)}^{t_{0}} \hat{D}(s) g(x(s)) d s-\int_{t_{0}}^{t}\left[\int_{u-\tau(u)}^{u} \hat{D}(s) g(x(s)) d s\right]-e^{-\int_{t_{0}}^{t} \hat{D}(s) d s} \hat{D}(u) d u \\
-\int_{t_{0}}^{t} e^{-\int_{u}^{t} \hat{D}(s) d s}\left[\int_{t_{0}}^{u} E(u, s) g(x(s-\tau(s))) d s\right] \hat{D}(u) d u
\end{gathered}
$$

This completes the proof of Lemma.

Theorem3.12. We suppose that the following assumptions hold:

(i) $\exists l>0 \mathrm{~S}$.T, the function $\mathrm{g}$ Satisfies the condition of Lipschitz on $[-l, l]$ and $\mathrm{g}$ is odd and is strictly increasing on $[-l, l]$ and $x-g(x)$ is no decreasing on $[-l, l]$.

(ii) $\exists \alpha \in(0,1)$, and the continuous function $a(t):[0, \infty) \rightarrow[0, \infty)$, S.T

$f_{1}(t, x, y) \geq a(t)$ for $t \geq 0, \quad x, y \in R$, and

$$
g(l)\left\{2 \sup _{t \geq 0} \int_{t}^{p(t)} \int_{0}^{\infty} e^{-\int_{u}^{t} a(v) d v} b(s) d w d s+2 \sup _{t \geq 0} \int_{0}^{t} \int_{t-s}^{\infty} e^{-\int_{s}^{w+s} a(v) d v} b(s) d w d s\right\}
$$


(iii) $\exists$ constants $a_{0}>0 \& Q>0$ S.T, for all $t \geq 0$, if $J>Q$ then

$$
\int_{t}^{t+J} a(v) d v \geq a_{0} J
$$

Then $\exists \delta \in(0, l)$ S.T for initial function $\psi:\left[m\left(t_{0}\right), t_{0}\right] \rightarrow R$ and $x^{\prime}\left(t_{0}\right)$ satisfying $\left|x^{\prime}\left(t_{0}\right)\right|+\|\psi\| \leq \delta$, there is the unique continuous function $x:\left[m\left(t_{0}\right), \infty\right) \rightarrow R$ satisfying $x(t)=\psi(t), t \in\left[m\left(t_{0}\right), t_{0}\right]$, is the solution of EQ.(15) on $\left[t_{0}, \infty\right)$. Furthermore, the zero solution of EQ.(15) is stable.

Proof. By choose ?: $\left[m\left(t_{0}\right), t_{0}\right] \rightarrow R$ and $x^{\prime}\left(t_{0}\right)$ such that

$$
\left(\mathcal{Q}+\frac{e^{-a_{0} \mathcal{Q}}}{a_{0}}\right)\left|x^{\prime}\left(t_{0}\right)\right|+\delta+g(\delta) \int_{t_{0}-\tau\left(t_{0}\right)}^{t_{0}} \hat{D}(u) d s \leq(1-\alpha) g(l) .
$$

By referring to the assumption $g(0)=0$ of the theory, it is clear that $g(l) \leq l$. Because $g(x)$ meets the Lipschitz condition in $[-l, l]$, and $g(x)$ is a continuous function in $[-l, l]$, there is a constant $\delta$ so that $\delta<l$.

Thus, from the statement $(P \phi)(t)$, it follows

$$
\begin{gathered}
|(P \varnothing)(t)| \leq \delta+\int_{t_{0}}^{t} e^{-\int_{t_{0}}^{t} \hat{D}(s) d s}\left|x^{\prime}\left(t_{0}\right)\right| e^{-\int_{t_{0}}^{u} A(s) d s} d u \\
+\int_{t_{0}}^{t} e^{-\int_{u}^{t} \hat{D}(s) d s} \hat{D}(u)(l-g(l)) d u+\int_{t_{0}}^{t} E(t, s) g(l) d s \\
+\int_{t-\tau(t)}^{t} \hat{D}(s) g(l) d s+\int_{t_{0}-\tau\left(t_{0}\right)}^{t_{0}} \hat{D}(s) g(\delta) d s \\
+\int_{t_{0}}^{t}\left[\int_{u-\tau(u)}^{u} \hat{D}(u) g(l) d s\right] e^{-\int_{u}^{t} \hat{D}(s) d s} \hat{D}(u) d u \\
+\int_{t_{0}}^{t} e^{-\int_{u}^{t} \hat{D}(s) d s\left[\int_{t_{0}}^{u} E(u, s) g(l) d s\right] \hat{D}(u) d u .}
\end{gathered}
$$

Moreover, according to theoretical assumptions, it follows

$$
\begin{gathered}
\int_{t_{0}}^{t} E(t, s)=\int_{t_{0}}^{t} \int_{t_{0}+t-s}^{\infty} e^{-\int_{s}^{u+s-t_{0}} A(v) d v} b(s) d u d s \\
=\int_{0}^{t} \int_{t-s}^{\infty} e^{-\int_{s}^{w+s} A(v) d v} b(s) d u d s \\
\leq \sup _{t \geq 0} \int_{0}^{t} \int_{t-s}^{\infty} e^{-\int_{s}^{u+s} A(v) d v} b(s) d u d s \\
\int_{t-\tau(t)}^{t} \hat{D}(s) d s=\int_{t-\tau(t)}^{t} \hat{D}(p(s)) d s=\int_{t-\tau(t)}^{t} \frac{\hat{D}(p(s))}{1-\tau^{\prime}(t)} d s \\
=\int_{t}^{p(t)} D(s) d s=\int_{t}^{p(t)} \int_{0}^{\infty} e^{-\int_{s}^{\tau+s} A(v) d v} b(s) d w d s \\
\leq \sup _{t \geq 0} \int_{t}^{p(t)} \int_{0}^{\infty} e^{-\int_{s}^{w+s} A(v) d v} b(s) d w d s, \\
g(l) \int_{t_{0}}^{t} E(t, s) d s+\int_{t_{0}}^{t}\left[\int_{u-\tau(u)}^{u} \hat{D}(u) g(l) d s\right] e^{-\int_{u}^{t} \hat{D}(s) d s} \hat{D}(u) d u
\end{gathered}
$$




$$
\begin{gathered}
+\int_{t-\tau(t)}^{t} \hat{D}(s) g(l) d s \int_{t_{0}}^{t}\left[\int_{u-\tau(u)}^{u} \hat{D}(u) g(l) d s\right] e^{-\int_{u}^{t} \hat{D}(s) d s} \hat{D}(u) d u \\
\leq g(l)\left\{\underset{t \geq 0}{2 \sup } \int_{t}^{p(t)} \int_{0}^{\infty} e^{-\int_{s}^{w+s} A(v) d v} b(s) d w d s+\underset{t \geq 0}{2 \sup } \int_{0}^{t} \int_{t-s}^{\infty} e^{-\int_{s}^{w+s} A(v) d v} b(s) d w d s\right\} \\
\leq \alpha g(l) .
\end{gathered}
$$

Therefore, we have

$$
\begin{gathered}
|(P \varnothing)(t)| \leq \delta+g(\delta) \int_{t_{0}-\tau\left(t_{0}\right)}^{t_{0}} \hat{D}(s) d s+(l-g(l))+\alpha g(l) \\
+\int_{t_{0}}^{t} e^{-\int_{t_{0}}^{t} \hat{D}(s) d s}\left|x^{\prime}\left(t_{0}\right)\right| e^{-\int_{t_{0}}^{u} A(s) d s} d u \\
\leq \delta+g(\delta) \int_{t_{0}-\tau\left(t_{0}\right)}^{t_{0}} \hat{D}(s) d s+(l-g(l))+\alpha g(l) \\
+\int_{t_{0}}^{t}\left|x^{\prime}\left(t_{0}\right)\right| e^{-\int_{t_{0}}^{u} A(s) d s} d u .
\end{gathered}
$$

By using the 3rd condition of the theorem, we're getting

$$
\begin{gathered}
\int_{t_{0}}^{t} e^{-\int_{t_{0}}^{u} A(s) d s} d u=\int_{t_{0}}^{t_{0+} \mathcal{Q}} e^{-\int_{t_{0}}^{u} A(s) d s} d u+\int_{t_{0+}}^{t} e^{-\int_{t_{0}}^{u} A(s) d s} d u \\
\leq \int_{t_{0}}^{t_{0+\mathcal{Q}}} d u+\int_{t_{0+}}^{t} e^{-a_{0}\left(u-t_{0}\right)} d u \leq \mathcal{Q}+\frac{e^{-a_{0} \mathcal{Q}}}{a_{0}}
\end{gathered}
$$

Hence,

$$
\begin{aligned}
|(P \varnothing)(t)| \leq & +g(\delta) \int_{t_{0}-\tau\left(t_{0}\right)}^{t_{0}} \hat{D}(s) d s+(l-g(l))+\alpha g(l) \\
& +\mathcal{Q}\left|x^{\prime}\left(t_{0}\right)\right|+\frac{e^{-a_{0}} \mathcal{Q}}{a_{0}}\left|x^{\prime}\left(t_{0}\right)\right|
\end{aligned}
$$

Since

$$
\left(\mathcal{Q}+\frac{e^{-a_{0} \mathcal{Q}}}{a_{0}}\right)\left|x^{\prime}\left(t_{0}\right)\right|+\delta+g(\delta) \int_{t_{0}-\tau\left(t_{0}\right)}^{t_{0}} \hat{D}(u) d s \leq(1-\alpha) g(l),
$$

then it follows that

$$
|(P \phi)(t)| \leq(\alpha-1) g(l)+l+(\alpha-1) g(l),
$$

so that

$$
|(P \phi)(t)| \leq l
$$

Thus, it is clear that if $t \in\left[m\left(t_{0}\right), t\right]$, then it can be seen that $\left(P_{2} \phi\right)(t)=\psi(t),\left|\left(P_{2} \phi\right)(t)\right| \leq l, t \in$ $\left[m\left(t_{0}\right), \infty\right)$. Therefore, $P \phi: S^{l} \rightarrow S^{l}$. Since $P$ is counteraction mapping $P$ have unique fixed point $x(l)$ S.T, $|x(t)| \leq l$.

From system (14),

$$
y(t)=x^{\prime}\left(t_{0}\right) e^{-\int_{t_{0}}^{t} A(s) d s}-\int_{t_{0}}^{t} e^{-\int_{u}^{t} A(s) d s} b(u) g(x(u-\tau(u))) d u,
$$

it can be obtained that

$$
|y(t)| \leq\left|x^{\prime}\left(t_{0}\right)\right|+M \int_{t_{0}}^{t} e^{-\int_{u}^{t} A(s) d s}|g(x(u-\tau(u)))| d u
$$




$$
\begin{gathered}
\leq \delta+M \int_{t_{0}}^{t} e^{-\int_{u}^{t} A(s) d s}|g(x(u-\tau(u)))| d u \\
\leq \delta+M \int_{t_{0}}^{t} e^{-\int_{u}^{t} A(s) d s}|x(u-\tau(u))| d u \\
\leq l\left(1+M \int_{t_{0}}^{t} e^{-\int_{u}^{t} A(s) d s}\right) d u \\
\leq l\left[2+M\left(\mathcal{Q}+\frac{e^{-a_{0} \mathcal{Q}}}{a_{0}}\right)\right]
\end{gathered}
$$

We can conclude that

$$
|x(t)|+|y(t)|<l\left[2+M\left(\mathcal{Q}+\frac{e^{-a_{0} \mathcal{Q}}}{a_{0}}\right)\right] .
$$

This finishes the proof of theorem.

\section{Conclusion}

A Linard equation type with multiple variable delays, EQ.(4), is considered. First, the stability, uniformly stability, bondedness and uniformly bondedness of solutions of this equation(4), have been discussed by the Lyapunov-Krasovskii functional approached. Later, the stability of the solutions of the same equation, when $\mathrm{p}(\mathrm{t})=0$ in equation (4) is studied by fixed point techniques. It is clear that the assumptions of Theorem (3.8) are completely different except the similarity of the assumption of Theorem (2.18) and the assumption of Theorem (3.10).Theoretical assumptions of theorem (3.8) are cleared, gracious and understandable. That is, Theorem (2.16) assumptions had quite simple shapes and can easily prove their applicability. However, the best of our knowledge, it may be difficult to say the same for the assumptions of Theorem (3.8). When we change EQ.(1) into a more complex form, finding an appropriate LyapunovKrasovskii functional, which gives meaningful results, may be difficulty. It should be noted construction or definition Lyapunov-Krasovskii functional Residue us the open problem in literature, till now. This fact shows the advantage of the fixed point theory over the Lyapunov's direct method in the special case. Further, in spite of the more effectiveness of the Lyapunov-Krasovskii works in natural and functional differential equations of the highest order, and the application of the fixed point theory of these equations is very difficult, and sometimes it may be impossible. By this fact, we mean that depending on the form and order of the differential equation given, sometimes the Lyapunov-Krasovskii functional approach has an advantage over the fixed point theory, and sometimes it is in the contrast. However, to date, the most effective way to study the qualitative behaviors of nonlinear differential equations in the higher ranks is still the Lyapunov second method. At the end, this method is old but yet active in scientific literature.

\section{References}

[1] L. C. Becker, T. A. Burton, Stability, fixed points and inverses of delays. Proc. Roy. Soc. Edinburgh Sect. A 136(2) (2006), 245-275. https:// doi.org/10.1017/s0308210500004546 1

[2] T. A. Burton, Stability and periodic solutions of ordinary and functional differential equations, Academic Press, Orlando, (1985). 1, 2

[3] T. A. Burton, Stability by fixed point theory or Liapunov theory: A comparison. Fixed Point Theory,4(1) (2003), 15-32. 1

[4] T. A. Burton, Fixed points and stability of a nonconvolution equation, Proc. Amer. Math. Soc. 132 (12) (2004), 36793687. 1

[5] T. A. Burton, Fixed points, stability and exact linearization, Nonlinear Anal. 61(5) (2005), 857-870. https: / / doi.org/10.1016/j.na.2005.01.079 1

[6] T. A. Burton, The case for stability by fixed point theory, Dyn. Contin. Discrete Impuls. Syst. Ser. A Math. Anal.13B (2006),253-263. 1

[7] T. A. Burton, Stability by fixed point theory for functional differential equations, Dover Publications, Inc., Mineola, NY, (2006). 1 
[8] T. A. Burton, T. Furumochi, Fixed points and problems in stability theory for ordinary and functional differential equations, Dynam. Systems Appl. 10(1) (2001), 89-116. 1

[9] T. A. Burton, R. H. Hering, Liapunov theory for functional-differential equations, 20th Midwest ODE Meeting (Iowa City, IA, 1991). Rocky Mountain J. Math. 24(1) (1994), 3-17. 1

[10] T. A. Burton, C. G. Townsend, On the generalized Linard equation with forcing function, J. Differential Equations, 4 (1968), 620-633. 1

[11] T. A. Burton, On the equation $x^{\prime \prime}+f(x) h^{\prime}(x) x^{\prime}+g(x)=e(x)$. Ann. Mat. Pura Appl. 4 (85) (1970), 277-285. 1

[12] T. Chen, W. Liu, Z. Hu, New results on the existence of periodic solutions for a higher-order Linard type $p$-Laplacian differential equation, Math. Methods Appl. Sci. 34(17) (2011), 2189-2196. https://doi.org/10.1002/mma.1516 1

[13] L. A. Cherkas, O. N. Malysheva, On the estimation of the number of limit cycles in Linard systems with a small parameter, Differ. Uravn.47(2) (2011), 225-230; translation in Differ. Equ. 47(2)(2011), 224-230. 1

[14] X. Hou, Z. Wu, Existence and uniqueness of periodic solutions for a kind of Linard equation with multiple deviating arguments, J. Appl. Math. Comput. 38(1-2) (2012), 181-193. https://doi.org/10.1007/s12190-010-0472-x 1

[15] Z. Jin, Boundedness and convergence of solutions of a second-order nonlinear differential system, J. Math. Anal. Appl.256(2) (2001), 360-374. https://doi.org/10.1006/jmaa.2000.7056 1

[16] S. Jitsuro, A.Yusuke, Global asymptotic stability of non-autonomous systems of Lienard type, J. Math. Anal. Appl., 289(2) (2004), 673-690. https://doi.org/10.1016/j.jmaa.2003.09.023 1

[17] N. N. Krasovskii, Stability of motion. Applications of Lyapunov's second method to differential systems and equations with delay, Translated by J. L. Brenner Stanford University Press, Stanford, Calif. 1963. 1

[18] B. Liu, L.Huang, Boundedness of solutions for a class of retarded Linard equation, J. Math. Anal. Appl. 286 (2) (2003), 422-434. https:/ / doi.org/10.1016/s0022-247x(03)00455-4 1

[19] D. Pi, Study the stability of solutions of functional differential equations via fixed points, Nonlinear Anal. 74(2) (2011), 639-651. https:/ / doi.org/10.1016/j.na.2010.09.018 1, 3

[20] J. Sugie, Y. Amano, Global asymptotic stability of non-autonomous systems of Linard type, J. Math. Anal. Appl. 289(2) (2004), 673-690. 1

[21] C. Tun, Some new stability and boundedness results of solutions of Linard type equations with deviating argument, Nonlinear Anal. Hybrid Syst. 4(1) (2010), 85-91. https:/ / doi.org/10.1016/j.nahs.2009.08.002 1

[22] C. Tun, Boundedness analysis for certain two-dimensional differential systems via a Lyapunov approach.Bull, Math.Soc.Sci. Math. Roumanie(N.S.), 53(101) (2010), 61-68. 1

[23] C. Tun, S. A. Mohammed, On The Stability and Instability of Functional Volterra Integro-Differential Equations of First Order, Bulletin of Mathematical Analysis and Applications,9(1) (2017), 151-160. 1

[24] C. Tun, A note on boundedness of solutions to a class of non-autonomous differential equations of second order, Appl. Anal. Discrete Math.4(2) (2010), 361-372. https:/ / doi.org/10.2298/aadm100601026t 1

[25] C. Tun, New stability and boundedness results of solutions of Linard type equations with multiple deviating arguments, J. Contemp. Math. Anal. 45(3) (2010), 47-56. 1

[26] C. Tun, On the stability and boundedness of solutions of a class of no autonomous differential equations of second order with multiple deviating arguments, Afr. Mat. 23(2) (2012), 249-259. https:// doi.org/10.1007/s13370-011-0033-y 1

[27] C. Tun, Stability and uniform boundedness results for non-autonomous Linard-type equations with a variable deviating argument, Acta. Math. Vietnam. 37(3) (2012), 311-326. 1

[28] C. Tun, Stability to vector Lienard equation with constant deviating argument, Nonlinear Dynam.73(3) (2013), 12451251. https: / / doi.org/10.1007/s11071-012-0704-8 1

[29] C. Tun, $A$ note on the bounded solutions to $x^{\prime \prime}+c\left(t, x, x^{\prime}\right)+q(t) b(x)=f(t)$,Appl. Math. Inf. Sci. (AMIS). 8(1) ( 2014), 393-399. 1

[30] C. Tun, S. A. Mohammed, On the qualitative properties of differential equations of third order with retarded argument, Proyecciones J. of Math. 33(3) (2014), 325-347. https:/ / doi.org/10.4067/s0716-09172014000300007 1

[31] C. Tun, S. A. Mohammed, New results on exponential stability of nonlinear Volterra integro-Differential equations with constant time-lag Proyecciones, Journal of Mathematics. (2017). 1

[32] C. Tun, S. A. Mohammed, A remark on the stability and boundedness criteria in retarded Volterra integro-differential equations, J. of the Egy. , (2017). https://doi.org/10.1016/j.joems.2017.05.001 1

[33] C. Tun, S. A. Mohammed, On the stability and uniform stability of retarded integro-differential equations, Alexandria Engineering J., 57(2018), 3501-3507. https:/ / doi.org/10.1016/j.aej.2017.11.018 1

[34] C. Tun, S. A. Mohammed, Uniformly Boundedness in Nonlinear Volterra Integro-Differential Equations with Delay, J. of Appl. Nonlinear Dynamics, 8(2)(2019) 279-290. https:/ / doi.org/10.5890/jand.2019.06.010 1

[35] C. Tun, E. Tun, On the asymptotic behavior of solutions of certain second-order differential equations, J. Franklin Inst. 344(5) (2007), 391-398. 1

[36] N. Valds, Boundedness and global asymptotic stability of the forced Linard equation, Rev. Un. Mat. Argentina, 41(4) (2000), 47-59. 1

[37] T. Yoshizawa, Stability theory by Liapunov's second method, Publications of the Mathematical Society of Japan, no. 9, The Mathematical Society of Japan, Tokyo (1966). 1, 2

[38] B. Zhang, Fixed points and stability in differential equations with variable delays, Nonlinear Anal., 63 (2005),233-242. https://doi.org/10.1016/j.na.2005.02.081 1

[39] X. Zhou, W. Jiang, W., Stability and boundedness of retarded Linard-type equation, Chinese Quart. J. Math. 18(1) (2003), 
7-12. 1

[40] Y. Zhang, Eventually vanished solutions of a forced Linard system, Appl. Math. Mech. 30(10) (2009), $1335-1344$. https:/ / doi.org/10.1007/s10483-009-1013-6 1 